\title{
C-Shaped Scotoma after Complicated Cesarean Section: A Case of Acute Macular Neuroretinopathy
}

\author{
Teresa Rauchegger ${ }^{a}$ Georgios Blatsios ${ }^{a} \quad$ Gertrud Haas ${ }^{a} \quad$ Günther Putz ${ }^{b}$ \\ Claus Zehetner ${ }^{\mathrm{a}}$ \\ aDepartment of Ophthalmology and Optometry, Medical University Innsbruck, \\ Innsbruck, Austria; bepartment of Anesthesiology and Intensive Care, Medical University \\ Innsbruck, Innsbruck, Austria
}

\section{Keywords}

Acute macular neuroretinopathy · Epinephrine - Cesarean section - Retinal microvasculature . Spectral-domain optical coherence tomography

\begin{abstract}
Acute macular neuroretinopathy (AMN) is a rare retinal disease that produces transient or permanent visual impairment and occurs predominantly in young, Caucasian women of childbearing age. It is often characterized by wedge-like macular lesions. Although the cause of AMN is unknown, recent research suggests a microvascular etiology. Various vascular pathologies, including post-viral illness, oral contraceptives, and use of vasoconstrictive agents, have been associated with AMN. We present a case of a woman with C-shaped visual field defects in both eyes after inadvertent exposure to intravenous high-dose epinephrine during onset of spinal anesthesia. At present, only 8 cases of AMN after exposure to epinephrine have been described in literature. To our knowledge, this is the first case of AMN that presented following epinephrine injection during childbirth.




\section{Introduction}

Acute macular neuroretinopathy (AMN) is a rare retinal condition that was first described by Bos and Deutman [1] in 1975. AMN occurs predominantly in young, Caucasian women of childbearing age [2].

The onset of the disease is characterized by photopsia and paracentral scotoma associated with mild visual impairment. The acute onset of reddish brown parafoveal macular lesions is a characteristic feature of AMN. The configuration of these lesions is described as wedge shaped, petaloid or tear-drop shaped, and they generally point toward the fovea [3]. In $50 \%$ of the cases, the disease occurs bilaterally [2]. Although the exact pathophysiology of AMN remains unknown, a vascular pathology has been proposed. Several risk factors have been associated with the onset of the disease, including nonspecific flu-like symptoms or fever, oral contraceptives, contact with vasoconstrictive agents, trauma, or hypovolemic shock. At present, 8 cases of AMN after exposure to epinephrine have been described in the literature [2].

Currently, there is no known therapy for AMN, and the prognosis is variable. About $50 \%$ of the patients suffer from persistent visual-field defects [2].

We present a case of a 26-year old Caucasian female who suffered from AMN after an emergency cesarean section with intravenous epinephrine injection.

\section{Case Presentation}

A 26-year-old multigravida woman was referred to an ophthalmological examination by the department of gynecology. She described a persisting, flickering, c-shaped defect of the visual field in both eyes. The patient reported that her vision was impaired immediately after an emergency cesarean section.

During onset of spinal anesthesia, the patient developed bradycardia and hypotension treated with intravenous ephedrine (10 mg) and inadvertently epinephrine $(1 \mathrm{mg})$ instead of atropine. As a result, life-threatening hypertensive crisis (blood pressure $>300 / 200 \mathrm{~mm} \mathrm{Hg}$ ), cardiac arrhythmia (polytope ventricular extrasystoles), and angina developed. After immediate induction of general anesthesia, she gave birth to a healthy boy. The patient recalled that her vision was impaired immediately after surgery. She described a flickering, c-shaped defect of the visual field in both eyes. Her medical history was unremarkable, and there was no family history of eye disease.

During ophthalmologic examination in bed at the obstetric ward 1 day after the cesarean section, uncorrected visual acuity showed 20/25 Snellen. Examination with a head-worn indirect ophthalmoscope was unremarkable, and a follow-up examination was recommended.

One month later, the patient was seen at the Ophthalmology Department because of persisting c-shaped scotoma in both eyes. Upon examination, her best-corrected visual acuity was 20/20 Snellen. Pupillary reflexes were normal without a relative afferent pupillary defect. Slitlamp examination (including intraocular pressures) and funduscopic examination were all unremarkable (Fig. 1a). Automated visual-field testing revealed paracentral visual-field defects (Fig. 1b).

Spectral-domain optical coherence tomography (SD-OCT) of the macula showed parafoveal disruptions of the ellipsoid and interdigitation zone of the photoreceptor layers (Fig. 2, right). These lesions correlated with hyporeflective parafoveal wedge-shaped areas on nearinfrared imaging (Fig. 2, left). Full-field electroretinogram (ERG) was normal, but multifocal 
ERG clearly demonstrated diminished parafoveal amplitudes corresponding to the SD-OCT abnormality (Fig. 1c). Based on the typical OCT findings and the pathognomonic lesions in near-infrared imaging, the diagnosis of AMN caused by exposure to intravenous high-dose epinephrine during onset of spinal anesthesia was made.

Because there is no current treatment, we performed close follow-up. Twelve months after the cesarean section, she was evaluated with a visual acuity of 20/20 Snellen, but unchanged visual symptoms. SD-OCT and visual-field testing remained unchanged as well.

\section{Discussion}

AMN is a rare disease that affects the outer retinal layer. The most sensitive method to detect these changes is the combination of SD-OCT and near-infrared imaging. These diagnostic tools can even show lesions that are invisible to biomicroscopy fundus examination [2]. SDOCT demonstrates initial changes in the outer plexiform layer (OPL) and outer nuclear layer (ONL) of the macula, visible as a hyperreflective band, followed by disruption of the ellipsoid and interdigitation zone of the photoreceptor layers, similar to our case. This occurs due to a focal disruption of the junction between the outer segment of the photoreceptors and the retinal pigment epithelium [4].

The exact pathogenesis of these changes is unclear, but recent studies have proposed ischemia of the deep retinal capillary plexus (DCP) as a potential mechanism underlying the disease [5]. Epinephrine can cause vasoconstriction and a reduction of blood flow of the inner retinal layers [6]. This might cause an occlusion of the DCP, which is closely aligned with the OPL/ONL region. The OPL, consisting of photoreceptor axon terminals, lies directly adjacent to the DCP [7]. In support of this hypothesis, data from patients with diabetic retinopathy have shown an association between DCP ischemia and the disruption of photoreceptor layers, highlighting the contribution of the DCP to the oxygen requirements of photoreceptors and the outer retina [8]. Liu et al. [9] used projection-resolved OCT angiography to demonstrate compromised DCP flow in affected AMN areas at presentation with subsequent partial restoration after 3 weeks, supporting the hypothesis that ischemic DCP leads to reperfusion in later stages $[4,10]$.

Previous studies demonstrated temporal changes in the retinal microvasculature throughout pregnancy. Lupton et al. [11] showed an initial dilatation during the second trimester, followed by a slow constriction of retinal vessels during the remainder of pregnancy. We postulate that these hemodynamic changes during pregnancy have potentially amplified the vasoconstrictive effect of epinephrine on the retinal microvasculature supplying the photoreceptor layers.

In conclusion, this case illustrates the occurrence of AMN associated with inadvertent systemic epinephrine injection in a woman during delivery. AMN is a rare disorder characterized by the acute onset of paracentral visual-field defects that occur mostly in young women of childbearing age. The pathogenesis is potentially associated with the vasoconstrictive effect of epinephrine on the retinal microvasculature and consecutive ischemic degradation of the neurosensory retina. The most sensitive diagnostic tools are SD-OCT and near-infrared imaging, revealing even lesions that are invisible to biomicroscopy fundus examination. This emphasizes the importance of modern imaging technology in rare entities such as AMN. This report is important to raise awareness among clinicians of the potential for developing AMN in patients after epinephrine injection, especially if they complain about visual field defects. 


\section{Statement of Ethics}

This case report was conducted in accordance with good clinical practice. We obtained written consent of the patient to use her data and images. There are no ethical conflicts to disclose.

\section{Disclosure Statement}

There are no conflicts of interest to declare.

\section{Funding Sources}

The authors received no specific funding for this work.

\section{Author Contributions}

Teresa Rauchegger: conception of the work, draft of the paper, final approval of the version to be published, and agreement to be accountable for all aspects of the work.

Georgios Blatsios, Gertrud Haas, Günther Putz: analysis of the data for the work, critical revision of the paper, final approval of the version to be published, and agreement to be accountable for all aspects of the work.

Claus Zehetner: substantial contributions to the conception of the work, analysis of the data for the work, critical revision of the paper, final approval of the version to be published, and agreement to be accountable for all aspects of the work.

\section{References}

1 Bos PJ, Deutman AF. Acute macular neuroretinopathy. Am J Ophthalmol. 1975 Oct;80(4):573-84.

2 Bhavsar KV, Lin S, Rahimy E, Joseph A, Freund KB, Sarraf D, et al. Acute macular neuroretinopathy: A comprehensive review of the literature. Surv Ophthalmol. 2016 Sep-Oct;61(5):538-65.

3 Turbeville SD, Cowan LD, Gass JD. Acute macular neuroretinopathy: a review of the literature. Surv Ophthalmol. 2003 Jan-Feb;48(1):1-11.

4 Fawzi AA, Pappuru RR, Sarraf D, Le PP, McCannel CA, Sobrin L, et al. Acute macular neuroretinopathy: longterm insights revealed by multimodal imaging. Retina. 2012 Sep;32(8):1500-13.

5 Rahimy E, Sarraf D. Paracentral acute middle maculopathy spectral-domain optical coherence tomography feature of deep capillary ischemia. Curr Opin Ophthalmol. 2014 May;25(3):207-12.

6 Aziz HA, Kheir WJ, Young RC, Isom RF, Dubovy SR. Acute macular neuroretinopathy: a case report and review of the literature, 2002-2012. Ophthalmic Surg Lasers Imaging Retina. 2015 Jan;46(1):114-24.

7 Sarraf D, Rahimy E, Fawzi AA, Sohn E, Barbazetto I, Zacks DN, et al. Paracentral acute middle maculopathy: a new variant of acute macular neuroretinopathy associated with retinal capillary ischemia. JAMA Ophthalmol. 2013 Oct;131(10):1275-87.

8 Scarinci F, Nesper PL, Fawzi AA. Deep Retinal Capillary Nonperfusion Is Associated With Photoreceptor Disruption in Diabetic Macular Ischemia. Am J Ophthalmol. 2016 Aug;168:129-38.

9 Liu JC, Nesper PL, Fawzi AA, Gill MK. Acute macular neuroretinopathy associated with influenza vaccination with decreased flow at the deep capillary plexus on OCT angiography. Am J Ophthalmol Case Rep. 2018 Feb;10:96-100.

10 Ashraf M, Goldstein D, Fawzi A. Optical Coherence Tomography Angiography: Potential Artifacts in Acute Macular Neuroretinopathy. JAMA Ophthalmol. 2017 Jun;135(6):675-6.

11 Lupton SJ, Chiu CL, Hodgson LA, Tooher J, Lujic S, Ogle R, et al. Temporal changes in retinal microvascular caliber and blood pressure during pregnancy. Hypertension. 2013 Apr;61(4):880-5. 


\section{Case Reports in Ophthalmology}

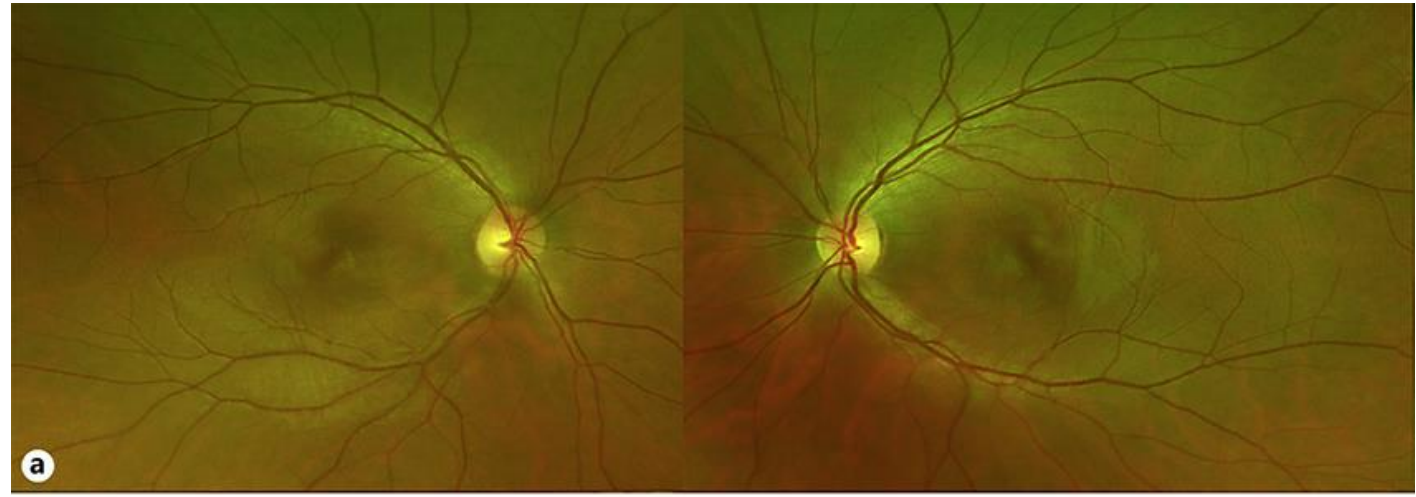

b
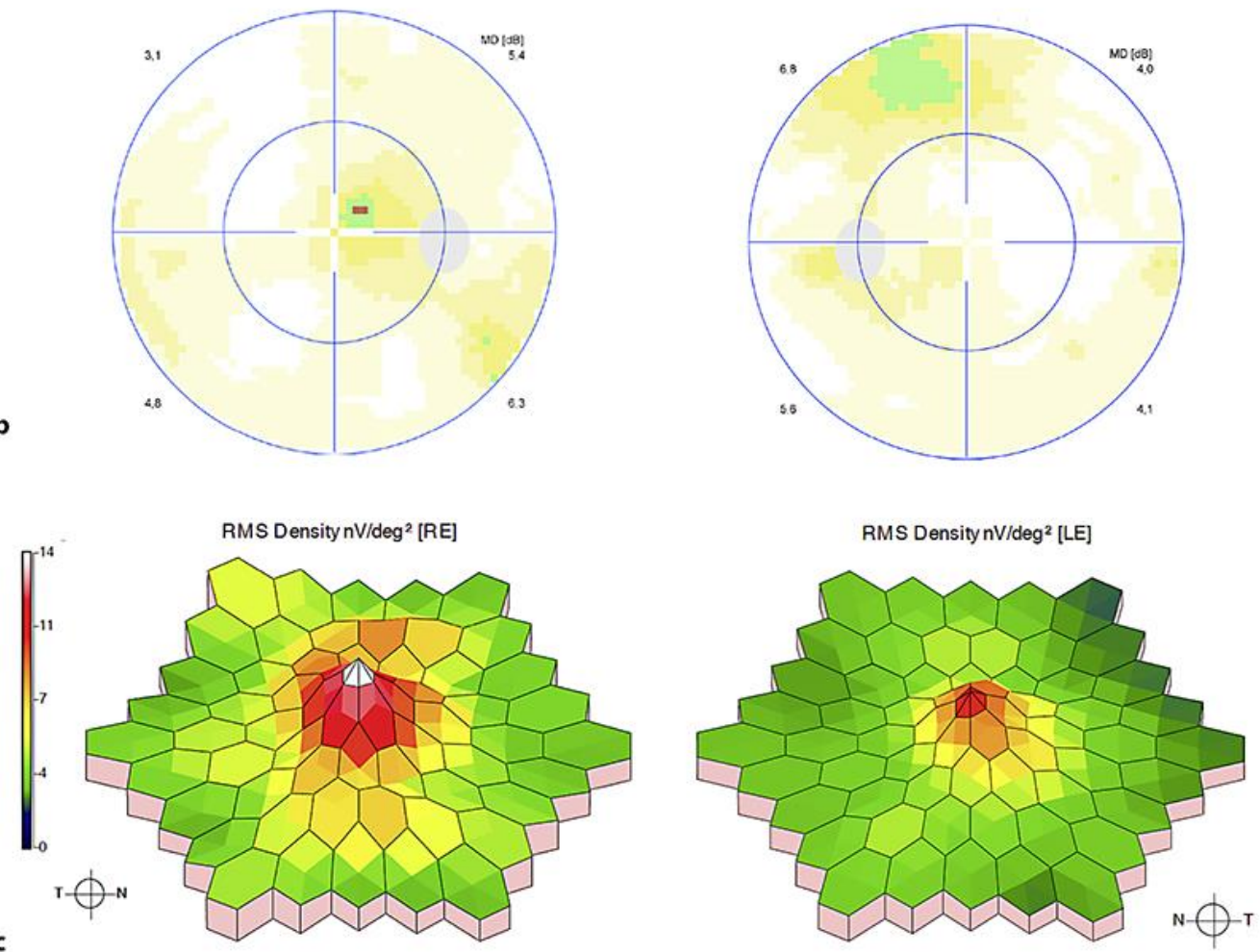

Fig. 1. a Scanning laser ophthalmoscopy images demonstrating a normal fundus. b Visual-field reports revealing paracentral visual-field defects, described as c-shaped by the patient. c Multifocal ERG demonstrating diminished parafoveal amplitudes. 


\section{Case Reports in Ophthalmology}

Case Rep Ophthalmol 2019;10:391-396

DOI: $10.1159 / 000504289$

(c) 2019 The Author(s). Published by S. Karger AG, Basel www.karger.com/cop

Rauchegger et al.: C-Shaped Scotoma after Complicated Cesarean Section
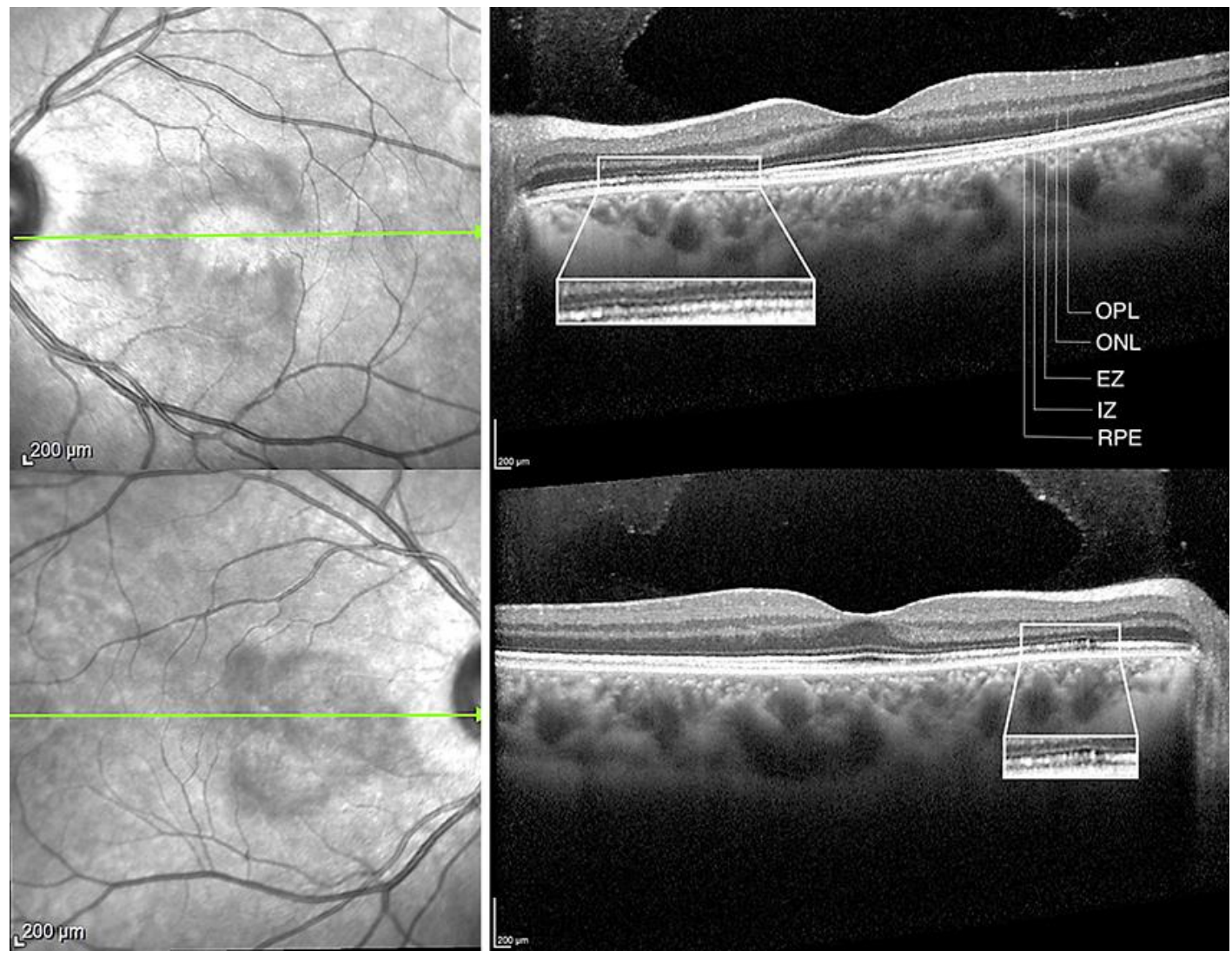

Fig. 2. Near-infrared imaging of the macula (left) and SD-OCT of the macula (right) showing parafoveal disruptions in the photoreceptor layers. 